\title{
Action Spectroscopy on Dense Samples of Photosynthetic Reaction Centers of Rhodobacter sphaeroides WT Based on Nanosecond Laser-Flash ${ }^{13}$ C Photo-CIDNP MAS NMR
}

\author{
Eugenio Daviso $\cdot$ Anna Diller $\cdot$ Peter Gast $\cdot$ A. Alia $\cdot$ \\ Johan Lugtenburg • Marc G. Müller • Jörg Matysik
}

Received: 2 September 2009/Published online: 3 December 2009

(c) The Author(s) 2009. This article is published with open access at Springerlink.com

\begin{abstract}
Photochemically induced dynamic nuclear polarization magic-angle spinning nuclear magnetic resonance (photo-CIDNP MAS NMR) allows for the investigation of the electronic structure of the photochemical machinery of photosynthetic reaction centers (RCs) at atomic resolution. For such experiments, either continuous radiation from white xenon lamps or green laser pulses are applied to optically dense samples. In order to explore their optical properties, optically thick samples of isolated and quinone-removed RCs of the purple bacteria of Rhodobacter sphaeroides wild type are studied by nanosecond laser-flash ${ }^{13} \mathrm{C}$ photo-CIDNP MAS NMR using excitation wavelengths between 720 and $940 \mathrm{~nm}$. Action spectra of both the transient nuclear polarization as well as the nuclear hyperpolarization, remaining in the electronic ground state at the end of the photocycle, are obtained. It is shown that the signal intensity is limited by the amount of accessible RCs and that the different mechanisms of the photo-CIDNP production rely on the same photophysical origin, which is the photocycle induced by one single photon.
\end{abstract}

\section{Introduction}

Action spectroscopy measures the response of the system to radiation depending on the wavelength and may allow for identifying the light-absorbing chromophore in a

E. Daviso - A. Diller · A. Alia $\cdot$ J. Lugtenburg $\cdot$ J. Matysik $(\bowtie)$

Institute of Chemistry, Leiden University, P.O. Box 9502, 2300 RA Leiden, The Netherlands

e-mail: j.matysik@chem.leidenuniv.nl

P. Gast

Institute of Physics, Leiden University, P.O. Box 9504, 2300 RA Leiden, The Netherlands

M. G. Müller

Max-Planck-Institut für Bioanorganische Chemie, Stiftstrasse 34-36,

45470 Mülheim an der Ruhr, Germany 
photo-process by comparing the efficiency of radiation of different wavelengths in driving the process [1-4]. This method has been introduced in 1928 as exakte Wirkungsspektrometrie by Otto Heinrich Warburg investigating the inhibition of the Atmungsferment (i.e., cytochrome- $c$ oxidase) by carbon monoxide, leading to the Nobel Prize in Medicine in 1931. Photosynthesis of plants and bacteria has been explored by action spectroscopy since the days of Warburg. In general, physiological parameters, such as adenosine triphosphate production, are observed to measure the action [5]. Alternatively, action spectroscopy in photosynthesis has been measured by optical, photothermal and photoacoustic methods [6-8].

Photosynthetic reaction centers (RCs) of purple bacteria of Rhodobacter (Rb.) sphaeroides wild type (WT) are well characterized [9]. The cofactor arrangement (Fig. 1) and the kinetics and spin dynamics of the electron transfer (Fig. 2) are well known. In its center, the primary electron donor $\mathrm{P}$, called the special pair, is composed of two Bacteriochlorophyll (BChl) cofactors $\mathrm{P}_{\mathrm{L}}$ and $\mathrm{P}_{\mathrm{M}}$. All cofactors are symmetrically arranged while the carotenoid alone breaks this symmetry. Upon illumination, an electron is transferred from the special pair, the primary electron donor $\mathrm{P}$, and a radical pair $\mathrm{P}^{+} \mathrm{BPhe}^{-}$is formed. It is born in a pure electronic singlet state $S_{0}$, which evolves coherently into a triplet state $T_{0}$, whilst the other two triplet states $\left(\mathrm{T}_{+1}\right.$ and $\left.\mathrm{T}_{-1}\right)$ are not populated at high magnetic fields. Several mechanisms have been identified transferring the electron polarization to nuclei. In continuousillumination photochemically induced dynamic nuclear polarization magic-angle spinning nuclear magnetic resonance (photo-CIDNP MAS NMR) experiments [1013], up to three mechanisms, called three-spin mixing (TSM) [14, 15], differential decay (DD) [16] and differential relaxation (DR) [17, 18] cause the build up of net photo-CIDNP. In laser-flash photo-CIDNP MAS NMR experiments [13, 19-21], transient nuclear polarization (TNP) is observed occurring selectively from the singlet branch of the radical-pair decay since the nuclear polarization of the triplet

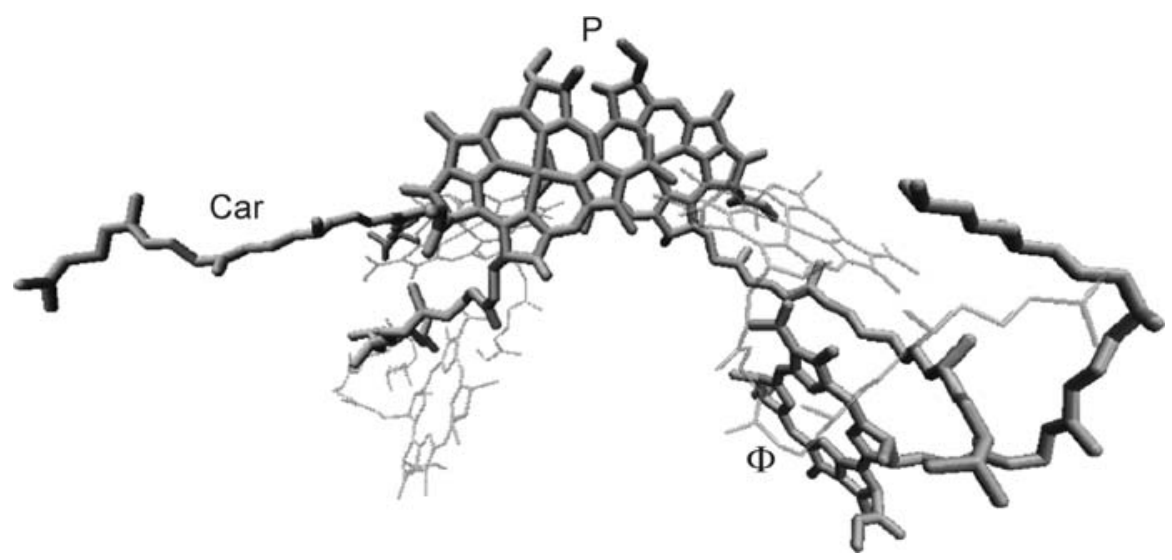

Fig. 1 Structural arrangement of cofactors in the RCs of Rb. sphaeroides WT. Cofactors involved into the spin dynamics of the primary radical-pair formation are: the primary electron donor $\mathrm{P}$ is formed by two bacteriochlorophyll $a$ molecules, $\mathrm{P}_{\mathrm{L}}$ (right) and $\mathrm{P}_{\mathrm{M}}$ (left), the (primary) electron acceptor $\Phi$, a bacteriopheophytin $a$ in the active L branch, and the carotenoid (Car). Other cofactors are shown in light grey (Protein Data Bank entry 1MX3) 


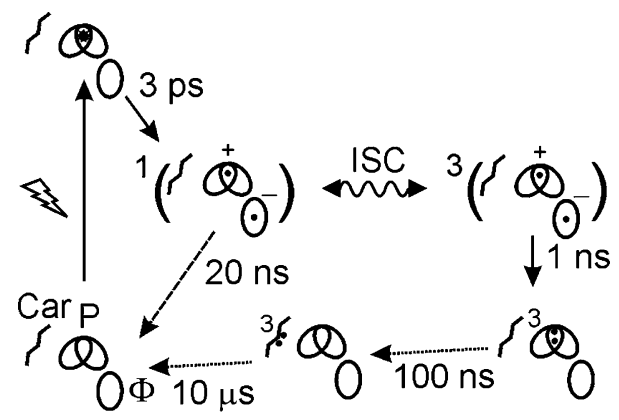

Fig. 2 Kinetics and spin dynamics of electron transport in quinone-depleted RCs of Rb. sphaeroides WT. After absorption of a photon, from the photochemically excited state of the primary donor $\mathrm{P}^{*}$, an electron is transferred to the primary acceptor $\Phi$, a bacteriopheophytin cofactor. This initial singlet radical pair ${ }^{1}\left(\mathrm{P}^{\bullet+} \Phi^{\bullet-}\right)$ is in a nonstationary state and highly electron polarized. An electron back-transfer leads to the electronic ground state (dashed arrow). Due to hyperfine interaction with nuclei, the singlet state of the radical pair evolves also into a triplet state ${ }^{3}\left(\mathrm{P}^{\bullet+} \Phi^{\bullet-}\right)$. Concomitant to this process of spin intersystem crossing (ISC), electron polarization is transferred to nuclei by the TSM and by the DD mechanisms. Net nuclear polarization is created by unbalancing the decay pathways of the singlet and the triplet radical pair (singlet and triplet branches). In addition, in time-resolved experiments TNP can be observed directly from the singlet decay channel because in the triplet decay pathway (triplet branch, dotted arrow), paramagnetic interaction of the triplet state of the nearby carotenoid, having a lifetime of 5 till $10 \mu \mathrm{s}$, leads to transiently obscured polarization (TOP)

branch is delayed. The occurrence of TNP can also be rationalized in terms of the classical radical-pair mechanism (RPM) [22, 23], which relies on diffusion and diminishes in steady-state experiments [20]. In WT samples, the TNP is caused by an effect called transiently obscuring polarization (TOP). It is caused by the strong paramagnetic interaction of the nearby carotenoid during its triplet state $\left({ }^{3} \mathrm{Car}\right)$, which transiently obscures the nuclear polarization of triplet decay branch $[20,21]$. After decay of the carotenoid triplet, this pool of nuclear polarization becomes visible in the NMR experiment again by extinguishing the opposite nuclear polarization of the singlet branch, and the TNP decays. The enhancement of signal intensity and selectivity of the nuclei allows for detailed studies on structure and spin dynamics of the photosynthetic cofactors active in the formation of the primary radical pair in various RCs [24-28].

Also the excitation transfer processes in RCs of $R b$. sphaeroides WT are well known. The four BChl cofactors and the two bacteriopheophytin (BPhe) cofactors are excitonically strongly coupled, allowing for fast and efficient excitation transfer towards $\mathrm{P}$ occurring on the time scale of some $100 \mathrm{fs}$ and with a quantum yield close to unity [29-33]. The carotenoid cofactor also functions as antenna, however, excitation transfer is slower ( $\sim 2 \mathrm{ps})$ and the quantum yield is lower $(\sim 75 \%)$ [34]. Hence, in optically thin samples, the action spectrum is almost identical with the absorption spectrum. In optically dense samples, shielding of the interior sample volume by the pigments in the front layers of the RCs, which are directly exposed to the laser pulses, blocks light transmission through the sample at the absorption frequencies and, therefore, the activation of the pigments in the inner parts of the sample. In addition, light scattering limits the penetration depth, especially of blue light. Under these conditions, the law of Lambert and Beer cannot be applied. 
In photosynthetic RC of $R b$. sphaeroides, bleaching is expected to cause an additional effect, since light absorption leads to the formation of radical pairs and these radical pairs have a different absorption spectrum. The main difference between the absorption spectrum of the $\mathrm{P}^{+} \mathrm{BChl}^{-}$radical pair and that of the electronic ground state is the total bleaching of the special-pair absorption at $860 \mathrm{~nm}$ once a radical pair is formed, while the other absorption bands undergo only little changes [35]. Hence, irradiation into the special-pair absorption may allow for substantial bleaching, especially under highly saturating laser pulses, which are used here and therefore result in a deeper penetration of the light into optically dense samples. Since NMR experiments with classical induction coils obtain the strongest signal from the center of the coil, an increase of penetration depth may have a strong effect on the obtained light-induced signals. For all photo-CIDNP MAS NMR experiments, as have been conducted till date, induction coils and samples in MAS rotors with diameters of 4 or $7 \mathrm{~mm}$ have been applied. In this study, we investigate the near-infrared (NIR) action spectrum of the solid-state photo-CIDNP effect in RCs of $R b$. sphaeroides WT in order to explore the way of light penetration into the samples.

\section{Materials and Methods}

\subsection{RC Preparation}

$\delta$-Aminolevulinic acid (ALA) is a precursor for biosynthetic tetrapyrrole synthesis. $R b$. sphaeroides WT was grown under anaerobic conditions in a medium containing $1.0 \mathrm{mM}$ $\left[4-{ }^{13} \mathrm{C}\right]-\mathrm{ALA}$, leading to a specific ${ }^{13} \mathrm{C}$ isotope label pattern on the $\mathrm{BChl}$ and BPhe cofactors (Fig. 3) [36]. The total incorporation $\left(P_{\text {tot }}\right)$ was calculated by the weighted sum:

Fig. 3 Bacteriochlorophyll $a$ (BChl) molecule with isotope label pattern biosynthetically introduced by feeding with $4-{ }^{13} \mathrm{C}$ - $\delta$-aminolevulinic acid (4-ALA). The bacteriopheophytin $a$ (BPhe) cofactors are labeled according to the same pattern

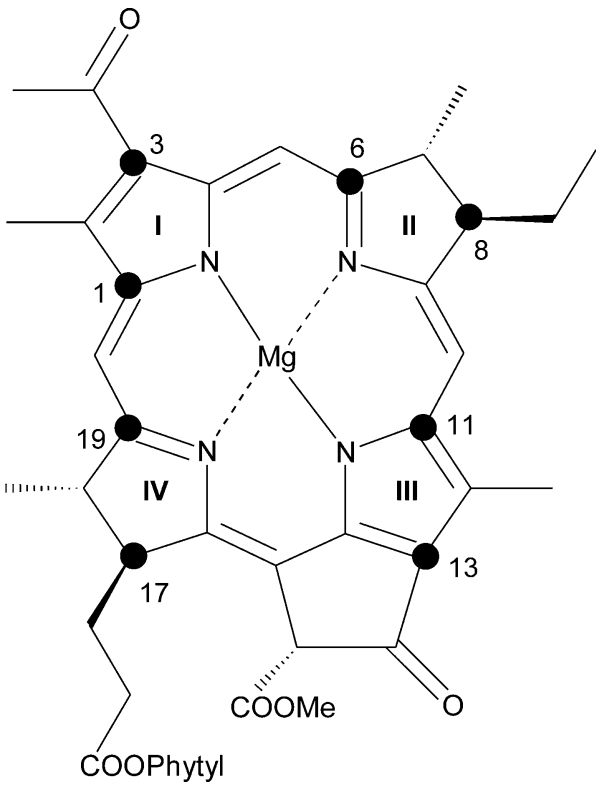




$$
P_{\text {tot }}=\sum_{n=0}^{8} \frac{n}{8} \times P_{n},
$$

where $n$ stands for the number of labels present in an isotopomer and $P_{n}$ is the corresponding fraction of labeled $\mathrm{BChl}$ estimated from the isotopic labeling pattern detected with the mass spectrometer [36]. The extent of ${ }^{13} \mathrm{C}$ incorporation has been determined by gas chromatography-mass spectrometry to be $60 \pm 5 \%$. The RCs were isolated by the procedure described in Ref. [36]. The quinone-depletion in the WT RC is reported in Ref. [11]. The samples were loaded into optically transparent colorless 4-mm sapphire rotors. The samples contained $10 \mathrm{mg}$ of RCs in a volume of about $70 \mu \mathrm{l}$ and scattered light significantly. The optical density was 1.35 at $865 \mathrm{~nm}$ after dilution by a factor 500. Using an extinction coefficient of $75 \mathrm{mM}^{-1} \mathrm{~cm}^{-1}$ [12], a sample concentration of $\sim 1 \mathrm{mM}$ has been calculated.

\subsection{Absorption spectrum}

For measuring of the absorption spectrum of isolated RCs of Rb. sphaeroides WT, $\sim 20 \mu \mathrm{g}$ of $\mathrm{RC}$ has been taken out of the MAS NMR rotor and diluted 500 times in tris-hydroxymethyl-aminomethane (Tris) and lauryldimethylamine- $N$-oxide (LDAO) buffer. The concentration was $\sim 0.02 \mathrm{mM}\left(\mathrm{OD}=1.35 \mathrm{~cm}^{-1}\right.$, extinction coefficient of $\left.75 \mathrm{mM}^{-1} \mathrm{~cm}^{-1}\right)$. The absorption spectrum has been measured at room temperature.

\subsection{Setup}

The nanosecond-laser flash photo-CIDNP setup has been described in detail elsewhere [19, 20]. Into this setup, an optical parametric oscillator (OPO 532/300, GWU-Lasertechnik, Erftstadt, Germany) tunable between 680 and $1050 \mathrm{~nm}$ has been incorporated. Laser power for all experiments has been $28.2 \mathrm{~mJ}$ and the beam diameter matched the inner diameter of the MAS rotor of about $3 \mathrm{~mm}$.

\subsection{MAS NMR measurements}

The NMR experiments were performed on an AV-200 NMR spectrometer (Bruker Biospin, Karlsruhe, Germany). Time-resolved nanosecond-flash photo-CIDNP MAS NMR measurements have been performed as described in Refs. [19-21] by changing the delay time between laser light pulse excitation and NMR detection systematically in three steps from 0 to 10 and $100 \mu$ s.

In all experiments, the signal has been detected with a polarization extinction technique allowing for fast scanning [19], using two-pulse phase modulation (TPPM) proton decoupling [37]. The cycle delay was $300 \mathrm{~ms}$. The temperature has been $233 \mathrm{~K}$ at a spinning frequency of $8 \mathrm{kHz}$. Artificial line broadening of $40 \mathrm{~Hz}$ has been applied. The number of scans was $120 \mathrm{k}$ per spectrum. The time-resolved photo-CIDNP MAS NMR spectra were referenced to the ${ }^{13} \mathrm{COOH}$ response of solid tyrosine $\cdot \mathrm{HCl}$ at $172.1 \mathrm{ppm}$. 


\section{Results and Discussion}

\subsection{NIR action spectroscopy with time-resolved photo-CIDNP MAS NMR}

The NIR absorption spectrum of diluted RCs of Rb. sphaeroides WT is shown as solid line in Fig. 4. The broad absorption band at longest wavelengths $(860 \mathrm{~nm})$ is the first electronic transition of the primary donor $\mathrm{P}$, which is of $\mathrm{Q}_{\mathrm{y}}$ character (for review, see Ref. [9]). The signals at 803 and $756 \mathrm{~nm}$ arise from the $\mathrm{Q}_{\mathrm{y}}$ transitions of the two accessory BChls and the BPhe cofactors, respectively. The weaker bands at 598 and $524 \mathrm{~nm}$ originate from the $\mathrm{Q}_{\mathrm{x}}$ transitions of the two accessory BChls and the BPhe cofactors, respectively. Features around $500 \mathrm{~nm}$ are caused by the carotenoid [33, 38]. The large signal around $400 \mathrm{~nm}$, which is not completely shown, is composed by the Soret bands of several cofactors.

Action spectroscopy requires a systematic variation of the excitation wavelength. Here, we report the dependence of the ${ }^{13} \mathrm{C}$ photo-CIDNP MAS NMR intensities on the excitation wavelength at investigating 15 excitations wavelengths in the range from 720 till $940 \mathrm{~nm}$. In Fig. 5, all the spectra have been collected using a delay time between the laser pulse and the NMR detection pulse of $100 \mu \mathrm{s}$. This set of experiments has been performed in order to understand the correlation between photo-CIDNP built up by TSM and DD as a function of the light wavelength. Trace A in Fig. 5 is obtained at $940 \mathrm{~nm}$, i.e., at wavelengths longer than the most redshifted absorption band. Trace B in Fig. 5 is measured at the maximum of the $\mathrm{Q}_{\mathrm{y}}$ absorption of the special pair $(860 \mathrm{~nm})$. Trace $C(820 \mathrm{~nm})$ in Fig. 5 is recorded between the absorption maxima of the special pair and the accessory BChls. Trace $\mathrm{D}(803 \mathrm{~nm})$ in Fig. 5 is obtained at the maximum of the accessory BChl absorption.

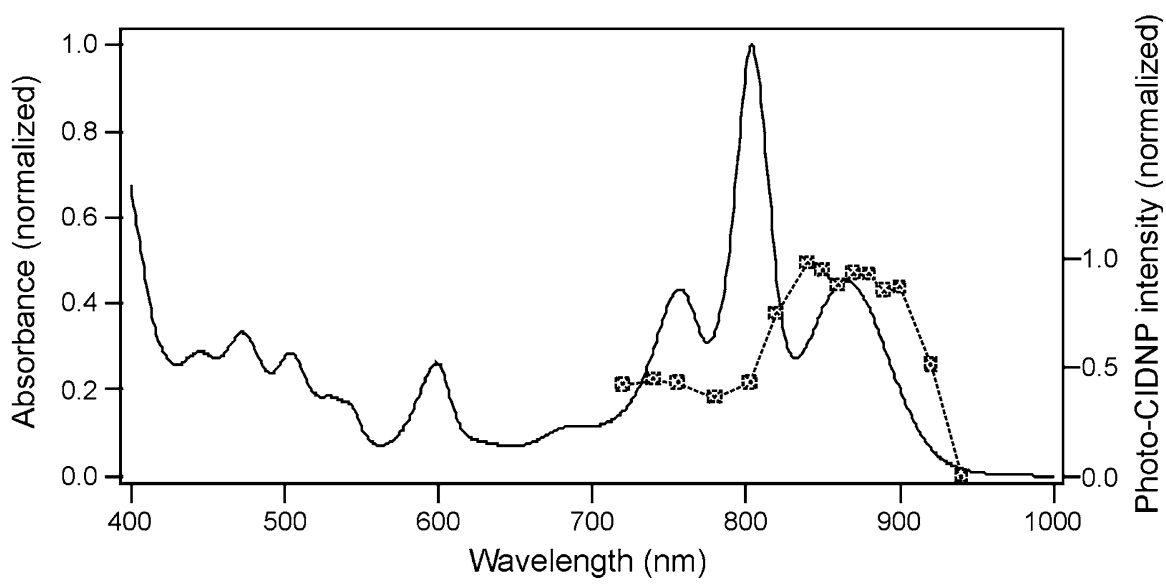

Fig. 4 The solid line is an ultraviolet-visible-NIR spectrum of purified RCs of Rhodobacter (Rb.) sphaeroides WT obtained at room temperature. The spectrum is normalized to the highest signal intensity at $860 \mathrm{~nm}$ (left scale). The peak at $860 \mathrm{~nm}$ is assigned to the bacteriochlorophylls of P. The peaks at 803 and $756 \mathrm{~nm}$ are assigned to the accessory bacteriochlorophylls and the bacteriopheophytins, respectively. The dotted line is the action spectrum of photo-CIDNP intensities of C-19 of $\mathrm{P}_{\mathrm{L}}(159.8 \mathrm{ppm})$ obtained $100 \mu \mathrm{s}$ after the laser flash and plotted as a function of the wavelength. The action spectrum is normalized to the highest signal intensity obtained with an excitation wavelength at $860 \mathrm{~nm}$ (right scale) 
Fig. $5{ }^{13} \mathrm{C}$ photo-CIDNP MAS NMR spectra of RCs of 4-ALA ${ }^{13} \mathrm{C}$-isotope labeled $R b$. sphaeroides WT collected using laser pulses at wavelengths of $940 \mathrm{~nm}(A), 860 \mathrm{~nm}(B)$, $820 \mathrm{~nm}(C), 803 \mathrm{~nm}(D)$, $780 \mathrm{~nm}(E), 756 \mathrm{~nm}(F)$, $720 \mathrm{~nm}(G)$. The absorption maxima of BPhe correspond to trace $F$, of the accessory BChls to trace $D$ and of the donor BChls to trace B. All the spectra have been collected at a magnetic field of $4.7 \mathrm{~T}$ and a temperature of $233 \mathrm{~K}$. The laser pulse length was $8 \mathrm{~ns}$ and the laser energy was $28 \mathrm{~mJ}$ in all experiments

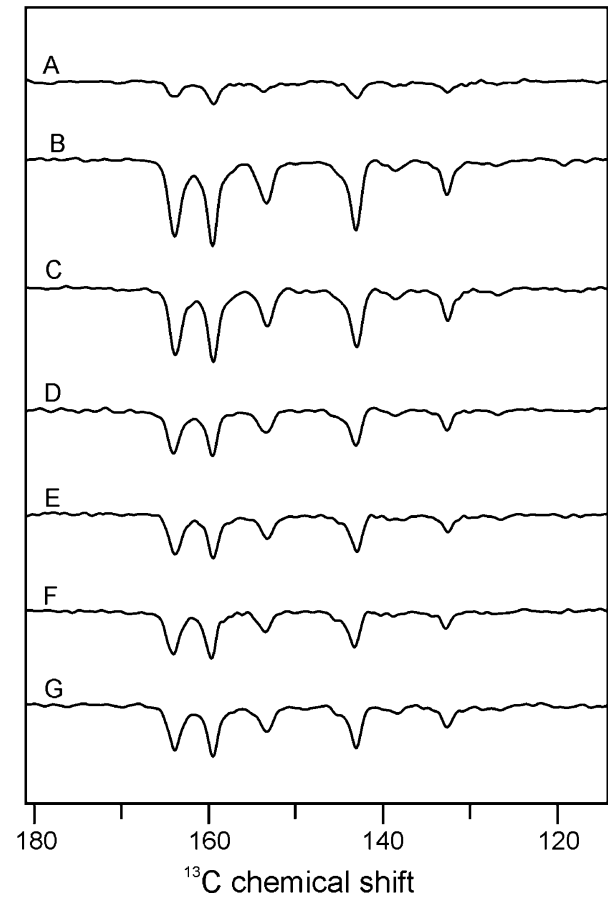

Trace E $(780 \mathrm{~nm})$ in Fig. 5 is measured in between the absorption maxima of the accessory BChls and the BPheos. Trace F $(756 \mathrm{~nm})$ in Fig. 5 is detected at the maximum of the BPheo absorption, and trace $\mathrm{G}(720 \mathrm{~nm})$ in Fig. 5 is registered in the low-absorbing region beyond the $\mathrm{Q}_{\mathrm{y}}$ transitions.

\subsection{Characteristics of action spectrum}

The dotted line in Fig. 4 shows the action spectrum as obtained for the C-19 resonance of the $\mathrm{P}_{\mathrm{L}}$ cofactor at $160.1 \mathrm{ppm}$ (Table 1). The photo-CIDNP intensities have been quantified for the two signals at $159.8 \mathrm{ppm}$ (assigned to $\mathrm{C} 19$ of $\mathrm{P}_{\mathrm{L}}$ ) and $164.0 \mathrm{ppm}$ (assigned to $\mathrm{C} 6$ of $\mathrm{P}_{\mathrm{L}}$ ). Since all light-induced signals are of the same photochemical origin, the ratio between them remains constant over the entire range of excitation frequencies. Only the value at $940 \mathrm{~nm}$ is different since the signal-tonoise ratio is too low for proper determination.

The action spectrum is essentially a flat but slightly tilted line with a single maximum at about $860 \mathrm{~nm}$. Once all the RCs are excited regardless of whether excitation happens to be in the absorption maximum of P860 or at the periphery, then the full photo-CIDNP signal amplitude is achieved. For this interpretation, further evidence is obtained by analysis of the shape of the broad action signal around $860 \mathrm{~nm}$. It shows a sharp increase at its flanks and constancy in its central area. Such behavior is typical for optical saturation of the signal. At conditions where the signal intensity is limited by the number of RCs which can be converted to a radical pair, the signal cannot further increase by increase of absorption. 
Table 1 Analysis of photo-CIDNP MAS NMR intensities measured at different excitation wavelengths $\lambda_{\text {exc }}$ and induced by different signal enhancement

\begin{tabular}{|c|c|c|c|c|c|}
\hline \multicolumn{3}{|l|}{ Data set } & \multirow{2}{*}{\multicolumn{2}{|c|}{$\frac{\text { Peak area }}{\text { TSM/DD } 100 \mu \mathrm{s}}$}} & \multirow[t]{3}{*}{ Ratio C19/C6 } \\
\hline \multirow[t]{2}{*}{ Number } & \multirow[t]{2}{*}{ Trace in Fig. 6} & \multirow[t]{2}{*}{$\lambda_{\text {exc }}(\mathrm{nm})$} & & & \\
\hline & & & C6- $P_{L}$ & $\mathrm{C} 19-\mathrm{P}_{\mathrm{L}}$ & \\
\hline 1 & A & 940 & 0.17 & 0.24 & 1.42 \\
\hline 2 & & 920 & 0.56 & 0.65 & 1.16 \\
\hline 3 & & 900 & 0.83 & 0.92 & 1.11 \\
\hline 4 & & 890 & 0.82 & 0.97 & 1.19 \\
\hline 5 & & 880 & 0.94 & 0.99 & 1.05 \\
\hline 6 & & 870 & 0.88 & 1.00 & 1.14 \\
\hline 7 & B & 860 & 0.84 & 0.93 & 1.11 \\
\hline 8 & & 850 & 0.89 & 0.97 & 1.09 \\
\hline 9 & & 840 & 0.91 & 0.96 & 1.05 \\
\hline 10 & $\mathrm{C}$ & 820 & 0.74 & 0.78 & 1.06 \\
\hline 11 & $\mathrm{D}$ & 803 & 0.50 & 0.48 & 0.97 \\
\hline 12 & $\mathrm{E}$ & 780 & 0.45 & 0.46 & 1.04 \\
\hline 13 & $\mathrm{~F}$ & 756 & 0.50 & 0.49 & 0.99 \\
\hline 14 & & 740 & 0.51 & 0.53 & 1.03 \\
\hline 15 & G & 720 & 0.49 & 0.54 & 1.11 \\
\hline
\end{tabular}

Signal enhancement by TSM and DD is measured by the intensity of the signal at $159.8 \mathrm{ppm}$ assigned to $\mathrm{C} 19$ of $\mathrm{P}_{\mathrm{L}}$ and by intensity of the signal at $164.0 \mathrm{ppm}$ assigned to $\mathrm{C} 6$ of $\mathrm{P}_{\mathrm{L}}$, measured at a delay time $\Delta$ of $100 \mu \mathrm{s}$

However, the maximum is clearly correlated with the absorption of $\mathrm{P}$, while the absorptions of the other bands do not cause any feature into the action spectrum. They can still contribute to the photo-CIDNP signal by energy transfer to P860 and subsequent charge separation. But unlike the $860 \mathrm{~nm}$ absorption band, these bands are not bleached, given the relatively long laser pulse width of $8 \mathrm{~ns}$ as compared to the internal energy transfer times of 100-200 fs. Thus, these bands result effectively in a kind of shielding of the lower sample layers and reduce the overall amount of radical pairs being formed. Note that RCs in the front layers are already in the $\mathrm{P} 60^{+} \mathrm{BPhe}^{-}$state and cannot form a second radical pair during the laser pulse width again, and so it cannot increase the photo-CIDNP signal accordingly.

The featureless baseline in the regions of strong absorption demonstrates that the sample is in the regime of high optical density and corresponds with photo-CIDNP induction on the sample surface only.

While there is no characteristic feature in the photo-CIDNP action spectrum around the absorption bands of the accessory BChls and the BPheos, the absorption of the special pair induces a clear effect on the action spectrum. The general appearance of the action spectrum is clearly distinguished from the absorption spectrum of the electronic ground state. For an optically thin sample, a coincidence of both types of spectra would be expected. The features of the action spectrum indeed agree with the absorption spectrum of the primary radical pair transient, 
showing strong bleaching around $860 \mathrm{~nm}$ [35], while there is no significant change of photo-CIDNP intensities for the other cofactors. Therefore, we conclude that the action spectrum mainly reflects the transient bleaching of the sample which allows for deeper penetration of light. Hence, it is the strength of its bleaching which distinguishes $\mathrm{P}$ in the long living radical pair state from the other cofactors and allows for its direct excitation under conditions of optically dense samples. This finding is in line with the strong photo-CIDNP MAS NMR signal obtained with 532-nm excitation [19-21], occurring at an absorption wavelength having low absorption and therefore great penetration depth.

\subsection{Ratio of TNP- to TSM/DD-induced nuclear polarization}

Figure 6 shows time-resolved photo-CIDNP MAS NMR spectra obtained at three selected excitation wavelengths (traces A-C) with three delay times between the laser pulse and the NMR detection pulse of 0,10 and $100 \mu$ s (spectra shown in black, grey and light grey, respectively). Comparing the three traces, the spectral

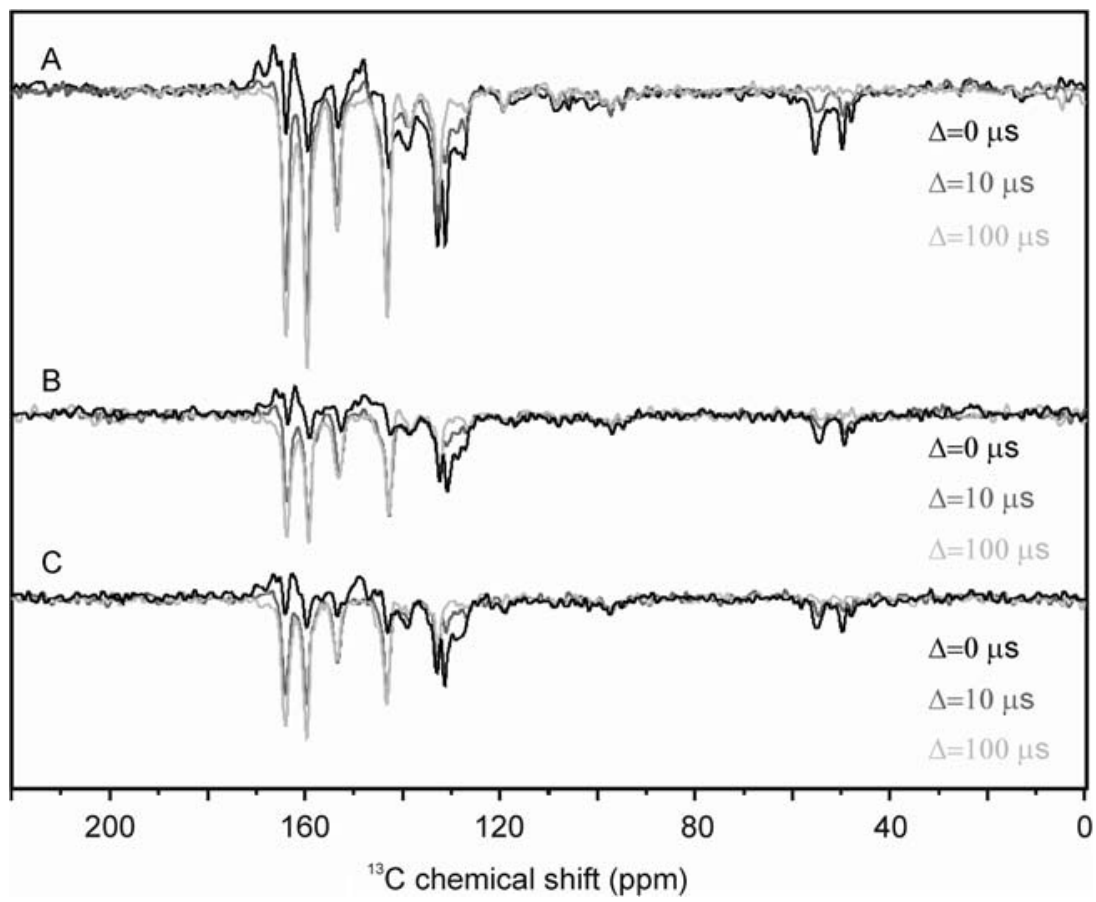

Fig. $6{ }^{13} \mathrm{C}$ photo-CIDNP MAS NMR spectra of RCs of 4-ALA ${ }^{13} \mathrm{C}$-isotope labeled Rb. sphaeroides WT collected using laser pulses at wavelengths of $860 \mathrm{~nm}(A), 803 \mathrm{~nm}(B)$ and $756 \mathrm{~nm}(C)$, respectively. The absorption maxima of the donor BChls correspond to trace $A$, of the accessory BChls to trace $B$ and of the BPhe to trace $C$. Traces $A, B$ and $C$ show the superposition of three spectra having different time delays between the light pulse and the NMR detection pulse (black $0 \mu \mathrm{s}$, grey $10 \mu \mathrm{s}$, and light grey $100 \mu \mathrm{s})$. All the spectra have been collected at a magnetic field of $4.7 \mathrm{~T}$ and a temperature of $233 \mathrm{~K}$. The laser pulse length was $8 \mathrm{~ns}$ and the laser power was $28.2 \mathrm{~mJ}$ in all experiments 
Table 2 Analysis of photo-CIDNP MAS NMR intensities measured at different excitation wavelengths $\lambda_{\text {exc }}$ and induced by different signal enhancement mechanisms

\begin{tabular}{|c|c|c|c|c|c|}
\hline \multicolumn{3}{|l|}{ Data set } & \multicolumn{2}{|l|}{ Peak area } & \multirow{2}{*}{$\begin{array}{l}\text { Ratio TSM/DD } \\
\text { to TNP/TOP }\end{array}$} \\
\hline Number & Trace in Fig. 6 & $\lambda_{\text {exc }}(\mathrm{nm})$ & $\begin{array}{l}\text { TNP/TOP } \\
0 \mu \mathrm{s}\end{array}$ & $\begin{array}{l}\text { TSM/DD } \\
100 \mu \mathrm{s}\end{array}$ & \\
\hline 7 & A & 860 & -0.2 & -1.0 & 0.5 \\
\hline 11 & B & 803 & -0.1 & -0.5 & 0.5 \\
\hline 13 & $\mathrm{C}$ & 756 & -0.1 & -0.5 & 0.5 \\
\hline
\end{tabular}

Signal obscured by TOP is measured by the intensity of the signal at $159.8 \mathrm{ppm}$ assigned to C19 of $\mathrm{P}_{\mathrm{L}}$ measured at a delay time D of $0 \mu \mathrm{s}$. Signal enhancement via the TSM/DD mechanisms is measured by the intensity of the signal at $159.8 \mathrm{ppm}$ assigned to $\mathrm{C} 19$ of $\mathrm{P}_{\mathrm{L}}$ measured at a delay time $\mathrm{D}$ of $100 \mu \mathrm{s}$

evolution from 0 to $100 \mu$ s appears not to change, as suggested by the constancy of all spectral features and their intensity ratios.

While the spectra obtained at $0 \mu$ s (black) are mainly caused by the TNP due to broadening of the signals of the carbons of the donar by the nearby molecular triplet of the carotenoid (TOP). the spectrum at $100 \mu$ s (light grey) fully represents buildup by TSM and DD. For quantification of the contributions of the different enhancement mechanisms, we took the signal at $160.1 \mathrm{ppm}$, assigned to C19 of $\mathrm{P}_{\mathrm{L}}$ [20], measured at a delay time of $0 \mu$ s for quantification of the TNP/TOP effect. Signal enhancement via the TSM/DD mechanisms, also quantified by the signal intensity of the signal at $160.1 \mathrm{ppm}$, is measured at a delay time of $100 \mu \mathrm{s}$. Table 2 compiles the intensities of these signals and demonstrates that the ratio between TNP- and TSM/DD-induced mechanisms remains constant over the entire range of excitation wavelengths. Since the probability for two-photon processes has a different dependence on the gradient of the photon concentration in the sample than single-photon processes, this finding demonstrates that both TNP- and TSM/DDinduced nuclear polarization are originating from the same single photocycle.

Since TNP is observed in RCs of $R b$. sphaeroides WT for $10 \mu$ s [20, 21], i.e., the lifetime of the triplet state of the carotenoid, it has been proposed that TOP from the triplet decay branch allows for selective TNP observation of the nuclear spins from the singlet decay path. Since TOP is observed under both green and red/NIR excitation, it has been concluded that TNP in WT-RCs is not caused by direct light absorption of the carotenoid but by triplet transfer from $P$ to the carotenoid [20,21], implying that also the TOP-induced intensity relies on a single-photon process. Here, we provide evidence for this mechanism.

\section{Outlook}

A quantification of the effect, for example, according to the theory of Kubelka and Munk [39], may lead to an exact characterization of the optical properties of dense RC samples as well as of the sensitivity distribution within NMR coils. In particular, such knowledge would allow optimizing photo-CIDNP MAS NMR signals by using specific excitation wavelengths. 
Acknowledgments We would like to thank Profs. G. Jeschke and R. van Grondelle for helpful discussions. The help of F. Lefeber, K. B. Sai Sankar Gupta and K. Erkelens is gratefully acknowledged. J.M. thanks the generous support by the Netherlands Organization for Scientific Research (NWO) through a Vidi grant (700.53.423) as well as by the Leiden Institute of Chemistry (LIC). E.D. thanks European Molecular Biology Organization (EMBO) for a short-term fellowship (ASTF-131-2005).

Open Access This article is distributed under the terms of the Creative Commons Attribution Noncommercial License which permits any noncommercial use, distribution, and reproduction in any medium, provided the original author(s) and source are credited.

\section{References}

1. F. Haxo, Comparative Biochemistry of Photoreactive Systems (Academic Press, New York, 1960), pp. $339-376$

2. E.I. Rabinowitch, Govindjee, Photosynthesis (Wiley, New York, 1969), pp. 141-151

3. K.M. Hartmann, in Biophysik, vol. 2, eds. by W. Hoppe, W. Lohmann, H. Markl, H. Ziegler (Springer-Verlag, Berlin, 1982), pp. 122-152

4. L.O. Björn (ed.), Photobiology (Springer, New York, 2008)

5. G. Vanginkel, J.W. Kleinenhammans, Photochem. Photobiol. 31, 385-395 (1980)

6. J.B. Callis, M. Gouterman, W.W. Parson, Biochim. Biophys. Acta 267, 348-362 (1972)

7. S. Malkin, D. Cahen, Photochem. Photobiol. 29, 803-813 (1979)

8. R. Carpentier, B. Larue, R.M. Leblanc, J. Phys. 44, 355-360 (1983)

9. A.J. Hoff, J. Deisenhofer, Phys. Rep. 287, 2-247 (1997)

10. G. Jeschke, J. Matysik, Chem. Phys. 294, 239-255 (2003)

11. S. Prakash, Alia, P. Gast, H.J.M. de Groot, G. Jeschke, J. Matysik, J. Am. Chem. Soc. 127,1429014298 (2005)

12. S. Prakash, Alia, P. Gast, H.J.M. de Groot, J. Matysik, G. Jeschke, J. Am. Chem. Soc. 128, 1279412799 (2006)

13. E. Daviso, PhD Thesis, Leiden University, Leiden, 2008

14. G. Jeschke, J. Chem. Phys. 106, 10072-10086 (1997)

15. G. Jeschke, J. Am. Chem. Soc. 120, 4425-4429 (1998)

16. A. McDermott, M.G. Zysmilich, T. Polenova, Solid State Nucl. Magn. Reson. 11, 21-47 (1998)

17. R.A. Goldstein, S.G. Boxer, Biophys. J. 51, 937-946 (1987)

18. T. Polenova, A.E. McDermott, J. Phys. Chem. B 103, 535-548 (1999)

19. E. Daviso, A. Diller, A. Alia, J. Matysik, G. Jeschke, J. Magn. Reson. 190, 43-51 (2008)

20. E. Daviso, A. Alia, S. Prakash, A. Diller, P. Gast, J. Lugtenburg, J. Matysik, G. Jeschke, J. Phys. Chem. C 113, 10269-10278 (2009)

21. E. Daviso, S. Prakash, A. Alia, P. Gast, G. Jeschke, J. Matysik, Appl. Magn. Reson. (2009). doi: 10.1007/s00723-009-0050-2

22. G.L. Closs, L.E. Closs, J. Am. Chem. Soc. 91, 4549-4550 (1969)

23. R. Kaptein, J.L. Oosterhoff, Chem. Phys. Lett. 4, 195 (1969)

24. J. Matysik, Alia, P. Gast, H.J. van Gorkom, A.J. Hoff, H.J.M. de Groot, Proc. Natl. Acad. Sci. USA 97, 9865-9870 (2000)

25. Alia, E. Roy, P. Gast, H.J. van Gorkom, H.J.M. de Groot, G. Jeschke, J. Matysik, J. Am. Chem. Soc. 126, 12819-12826 (2004)

26. A. Diller, E. Roy, P. Gast, H.J. van Gorkom, H.J.M. de Groot, C. Glaubitz, G. Jeschke, J. Matysik, A. Alia, Proc. Natl. Acad. Sci. USA 104, 12767-12771 (2007)

27. E. Roy, Alia, P. Gast, H. van Gorkom, H.J.M. de Groot, G. Jeschke, J. Matysik, Biochim. Biophys. Acta 1767, 610-615 (2007)

28. A. Diller, P. Gast, G. Jeschke, A. Alia, J. Matysik, in Energy from the Sun, eds. by J. Allen, E. Gantt, J. Golbeck, B. Osmond (Springer, Dordrecht, 2008), pp. 10606-10614

29. E.W. Knapp, S.F. Fischer, W. Zinth, M. Sander, W. Kaiser, J. Deisenhofer, H. Michel, Proc. Natl. Acad. Sci. USA 82, 8463-8467 (1985)

30. A. Warshel, W.W. Parson, J. Am. Chem. Soc. 109, 6143-6152 (1987) 
31. E.M. Franken, J. Amesz, Biochim. Biophys. Acta 1319, 214-222 (1997)

32. B.A. King, T.B. McAnaney, A. de Winter, S.G. Boxer, J. Phys. Chem. B 104, 8895-8902 (2000)

33. V.I. Novoderezhkin, A.G. Yakovlev, R. van Grondelle, V.A. Shuvalov, J. Phys. Chem. B 108, 74457457 (2004)

34. S. Lin, E. Katilius, A.K.W. Taguchi, N.W. Woodbury, J. Phys. Chem. B 107, 14103-14108 (2003)

35. A.R. Holzwarth, M.G. Müller, Biochemistry 35, 11820-11831 (1996)

36. E.A.M. Schulten, J. Matysik, Alia, S. Kiihne, J. Raap, J. Lugtenburg, P. Gast, A.J. Hoff, H.J.M. de Groot, Biochemistry 41, 8708-8717 (2002)

37. A.E. Bennett, C.M. Rienstra, M. Auger, K.V. Lakshmi, R.G. Griffin, J. Chem. Phys. 103, 6951-6958 (1995)

38. Y. Kakitani, R. Fujii, Y. Koyama, H. Nagae, L. Walker, B. Salter, A. Angerhofer, Biochemistry 45, 2053-2062 (2006)

39. P. Kubelka , F. Munk, Z. Tech. Phys. 12, 593 (1931) 